\title{
LA STRAGE EVITABILE DEL GERMANWINGS
}

\author{
Nota del s.c. VITTORINO ANDREOLI (*)
}

(Adunanza dell'8 ottobre 2015)

SunTO. - Un co-pilota del volo Barcellona-Dusseldorf, rimasto solo in cabina perché il comandante si reca in toilette, si blocca dentro e attua il progetto premeditato di sbattere contro una parete dei Pirenei, portando a morte se stesso e 149 passeggeri. Il caso viene analizzato dallo psichiatra, leggendo la tragedia come espressione di un delirio di grandezza, che caratterizza la fase maniacale di un Disturbo Bipolare, Mostrando come pensare di avere in mano il destino di quelle persone, riporti alla condizione titanica di onnipotenza e allo stesso tempo ponendo la propria morte come condizione eroica per passare alla Storia. Un disturbo di cui soffriva da anni e che la Compagnia aerea ha ignorato.

ABSTRACT. - A co-pilot on the Barcellona-Dusseldorf fly, left alone in the cabin when the pilot went to the toilet, locked himself inside the pilots cabin and put into action a premeditatet plan of smashing into the side of the Pyrenees leading to rhe death of himself and 149 passengers. The case will be analyse by the psychiatrist, reading the tragedy as an expression of a delirium of greatness, which characterises the manic phase of a Bipolar Disorder. It shows how thinking that he had the destiny of those people in his hands brought about a titanic condition of omnipotence and at the same time the consideration that his own death would be heroic act that would go down in history. A disorder he had suffered for years and which the airline ignored.

${ }^{*}$ Psichiatra e scrittore, Istituto Lombardo Accademia di Scienze e Lettere, Milano, Italy, New York Academy of Sciences, USA.

E-mail: andreoli.vittorino@gmail.com 


\section{PREAMBOLO}

Mi occupo di matti, termine che uso con molto affetto. Generalmente i matti, i miei matti, quelli a cui sono molto legato, li conosco personalmente.

Il compito che mi è stato affidato è difficile, perché si tratta di un evento di una gravità enorme, compiuto da un uomo di cui ho potuto avere molte notizie, anche da colleghi tedeschi, ma manca la mia diretta conoscenza, e per uno psichiatra la relazione con la persona (il paziente) è essenziale per darne una valutazione precisa.

Ma prima di entrare nel caso, voglio aggiungere un'altra considerazione preliminare: il fatto è accaduto il 24 marzo di quest'anno (2015) ed eravamo tutti caduti in un vero stato di angoscia, perché si è trattato di una tragedia vera e propria.

Oggi non si rappresentano più le tragedie greche di Sofocle o di Euripide, ma questa lo è certamente se solo si ricorda che portò a morte 150 persone.

La considerazione riguarda la capacità che ha la nostra società di consumare le notizie: ho potuto constatare che ormai si tratta di un fatto così lontano che è come se non fosse accaduto. Colpisce la capacità che abbiamo di consumare le tragedie: un po' perché ogni giorno ne presenta una nuova, ma soprattutto per la capacità a trasformare rapidamente in banale anche dei fatti che sono molto importanti.

Questo atteggiamento non è certo quello dell'Istituto Lombardo che, pur passati alcuni mesi, ha deciso di ripensare, di riconsiderare un comportamento che ha a che fare con l'Uomo, che rimane il protagonista di questa tragedia (umana appunto).

\section{IL FATTO}

Non è inutile dedicare qualche minuto per rievocarlo.

La scena si svolge il 24 marzo su un aereo della Germanwings, una società di volo che la Lufthansa acquista nel 2009 per entrare nel settore dei voli low cost, e dunque in una cultura che premia sempre più il volare con poco, il che equivale il volare per tutti. Molte Compagnie di gran nome e di grande importanza hanno acquisito società minori per essere presenti in questo promettente mercato.

Non voglio trattare il tema dei voli ordinari economicamente, 
rispetto ai low cost, però devo fare un cenno sulle modalità con cui, per esempio, il personale opera in queste due diverse aree dell'economia del volo.

Le ore di volo giornaliere medie di un comandante dei low cost sono 11 e 12 minuti, mentre in una compagnia di linea, arrivano in genere a 9 ore e qualche minuto e giorni di lavoro sono alternati da giornate di riposo.

E' inoltre difficile prevedere il tempo di attività in una giornata, perché dipende dal traffico, dai turni, dalla disponibilità delle squadre di riserva.

Non c'è dubbio che l'economia dei low cost è possibile proprio per i ridotti costi del personale, ed è questa una cornice che va delineata, anche se noi parleremo in particolare del protagonista di questa tragedia, di un co-pilota, il "primo ufficiale", di un ruolo che prevede la responsabilità di gestire da solo l'aereo in assenza del comandante dalla cabina.

Il co-pilota in questo caso aveva accumulato solo 630 ore di volo: sono poche anche perché, come sappiamo, tutta la preparazione avviene a terra.

Anche in Italia, a Fiumicino c'è un grande ambiente per l'addestramento (a terra dunque) dei piloti, a cui ho avuto occasione di partecipare per un certo numero di volte, alcuni anni fa, quando erano in discussione le modalità di addestramento.

Il comandante dell'aereo invece di ore di volo ne aveva circa settemila, una dimensione notevolmente diversa.

Le compagnie low cost - anche questo è un dato generale - spesso prendono dei primi ufficiali, come si trattasse di rispettare una norma burocratica, e non sono molto attenti sulla loro preparazione, almeno non come lo sono le Compagnie nella scelta di ufficiali per voli di linea. E questo è un tema che va tenuto presente, sia pure in senso generale, perché altrimenti non capiremo bene l'accaduto. 9525.

Passiamo ora al 24 marzo e a quel volo della Germanwings, il

In cabina ci sono il comandante e il primo ufficiale, che quel giorno sono responsabili di un volo Düsseldorf-Barcellona.

Come avviene sovente, giunta a Barcellona la squadra ha un periodo di attesa, e poi opera sulla rotta inversa Barcellona-Düsseldorf.

Durante il volo da Düsseldorf, che parte alle 7.01 e arriva a Barcellona alle 8.57, all'andata dunque, il copilota ha avuto alcuni 
momenti di responsabilità totale di guida di quell'aereo, e analizzando bene le registrazioni della scatola nera avrebbe fatto un tentativo di calo di quota rapido e ingiustificato.

Il fatto lo si attribuì alla sua non grande esperienza, essendo lui relativamente giovane, e quindi non gli si diede grande importanza.

L'aereo arriva alle 8.57 a Barcellona, quindi riparte per Düsseldorf un po' in ritardo. Decolla infatti alle 10.01. L'arrivo a Düsseldorf è previsto per le 11.55. Alle 10.27 l'aereo, un Airbus A320-200 denominato Mannheim (in volo dal 29 novembre 1990 e consegnato alla Lufthansa il 5 febbraio 1991), raggiunge la quota di crociera, 11400 metri, con una velocità di $870 \mathrm{~km}$ all'ora.

Sull'aereo ci sono 145 passeggeri, tra cui una classe di scolari in gita premio. L'equipaggio è formato da 5 persone.

Ore 10.30: arrivato in quella che comunemente è chiamata la condizione di crociera, il comandante si alza e passa la responsabilità del volo al copilota perché deve andare in toilette.

Certo non si possono discutere i bisogni fisiologici, però, appena 30 minuti dal decollo, il bisogno sembra più una voglia di muoversi.

Il comandante esce.

Subito, dalla cabina di pilotaggio viene bloccata la porta. Il copilota blinda, come si dice, la cabina, nel senso che non può essere aperta dall'esterno.

Questa possibilità è stata introdotta dopo l'11 settembre 2001, dopo la tragedia delle Torri gemelle di New York. In quel caso i terroristi erano appunto entrati in cabina e con un'arma avevano obbligato il comandante ad andare contro uno dei due grattacieli.

Alle 10 e 35, (5 minuti dopo), il comandante bussa alla porta, ma il copilota, chiuso all'interno, non risponde.

Alle 10 e 41 scatta il fly monitor system, il quale automaticamente emette lo stato di allarme: è il computer di bordo che definisce di allarme la situazione, per il semplice motivo che avviene un grande salto di altitudine, poiché si passa in pochi minuti da 38 mila a 8 mila piedi.

Il copilota, il personaggio di cui parleremo, aveva inserito il pilota automatico, ma aveva modificato alcune condizioni di volo - questo per dire anche che aveva un piano preciso - quelle che impediscono che ci sia una caduta di quel tipo. A questo punto tutti si rendono conto come vanno le cose: i passeggeri sono disperati, cominciano a capire che l'aereo è fuori controllo: urla e pianti disperati rimbombano nell'aereo. 
Si sa tutto questo perché sono state recuperate le due scatole nere che riportano la registrazione degli avvenimenti.

Alle 10 e 47 l'aereo si trova davanti al massiccio noto come l'etoile. Tutti gli allarmi scattano, i sistemi automatici dei cosiddetti cervelli di bordo si mettono in azione, ma davanti ormai ci sono i Pirenei, le montagne.

E così dopo 40 minuti e 47 secondi dal decollo, con i passeggeri in stato di terrore, l'A320 sbatte contro la parete rocciosa. La velocità d'impatto è di $700 \mathrm{~km}$ all'ora, e questo permette anche di capire che non c'è nessuna possibilità di ritrovare il famoso sopravvissuto dei romanzi.

Alle 10 e 43 tutto è finito: tutti morti. E in questo dramma disumano, di ancor più funereo c'è che le persone non sono solo morte, ma sono state frammentate nell'impatto.

I gruppi di soccorso si affannano per arrivare presto nei pressi di Prads-Haute- Bleone preoccupati di salvare almeno la dignità di quei frammenti di corpi, perché in quella zona pirenaica vivono i lupi, e vogliono arrivare prima che quei frammenti di corpi diventino cibo.

\section{IL PROTAGONISTA}

Il protagonista è Andreas Gunt Lubitz. Ha 27 anni, aiutante del comandante, Patrick Sonderheimer, considerato un uomo di grande esperienza.

$\mathrm{Da}$ una delle scatole nere si rileva che mentre aspettavano di decollare da Barcellona si è svolto un colloquio tra i due, assolutamente privo di segni particolari.

Il signor Andreas Gunt è stato assunto - e questo è un altro punto importante - dalla Lufthansa nel 2013, e quindi era sotto la direzione generale di una delle compagnie aeree che gode nel mondo, godeva forse, della massima fiducia.

L'assunzione ha il significato di un riconoscimento per Lubitz che l'addestramento e le condizioni per svolgere quel lavoro sono assolutamente nella norma.

Qui si apre il problema di come si possa dare il titolo di copilota a uno che nel 2013 quando viene assunto, possedeva almeno la metà delle 630 ore di volo raggiunte nel 2015. Ma viene comunque assunto.

Una evenienza ancora più grave è che non viene dato nessun peso 
al fatto che nel 2008 questo giovane, ammesso ad un corso di addestramento, lo interrompe, e il motivo è "per disturbi psichici".

Dato questo bene documentato.

Si deve aggiungere che Lubitz aveva un deficit visivo che probabilmente mai gli avrebbe permesso di giungere al grado di comandante.

Si apre dunque il problema della valutazione, che esiste per tutte le professioni. Penso alle Forze dell'ordine, per citare un'organizzazione altrettanto importante, in cui la selezione significa consegnare un'arma di dotazione.

Si pone dunque un quesito chiave: se quella Compagnia (la Lufthansa) abbia potuto veramente valutare tutti i requisiti che si rendono necessari per una funzione così importante.

Io non so dare risposta in maniera definitiva, perché mi occupo soltanto di quella parte della storia che rientra nella mia professione, e parlerò cioè del protagonista.

Dall'analisi del suo computer è emerso che nei giorni precedenti la strage aveva svolto su internet una ricerca sul tema della blindatura delle cabine di pilotaggio. Pur essendo un pilota, ha voluto in qualche modo accertarsi, come fosse un dilettante, del funzionamento di questo sistema.

E anch'io, qualche giorno fa sono andato su internet a cercare dati sulla blindatura.

Ebbene, è facile scoprire i congegni meccanici o gli automatismi di tipo digitale che regolano questa possibilità.

Ma passiamo alla personalità di Andreas Gunt Lubitz.

Il primo elemento da tenere presente è l'aver interrotto l'addestramento con una diagnosi di depressione: questa era la valutazione certificata.

Il secondo elemento ci riporta alla sua ex fidanzata. Ha riferito testualmente: "un giorno, mi disse, farò un gesto che mi renderà noto a tutto il mondo".

Da una frase non possiamo dedurre molte cose, tuttavia è significativa.

Sappiamo che praticava sport atletici e che ha vinto alcune gare. Ogni giorno inoltre fa footing.

Questo impegno va sottolineato perché i depressi che fanno footing non sono molti, e se lo fanno quasi sempre sta a indicare che non sono (più) depressi. Emerge poi un dato che ha una certa forza: due 
giorni prima dell'evento gli viene fatto un certificato medico perché possa assentarsi dal lavoro, lui però non lo consegna. E' stato ritrovato, e risulta compilato da un medico di medicina generale (non da uno psichiatra).

Non c'è dubbio che siamo davanti a una personalità che alcune volte assume espressioni del tipo: diventerò importantissimo e che mostra di essere molto attivo nello sport, s'impegna, ha volontà.

E' emerso che tutta la sua storia è legata agli aerei. Comincia da ragazzo a costruire alianti e successivamente a voler diventare pilota. Il suo ideale è volare. In Germania l'hanno chiamato anche Icaro, però non è caduto da solo, si è tirato dietro 149 persone.

$\mathrm{Ma}$ a questo punto prima di definire in maniera conclusiva la sua personalità devo fare una premessa.

\section{VIOLENZA E DISTRUTTIVITÀ}

Noi oggi facciamo una grande distinzione tra violenza e distruttività. La violenza è certamente un agire contro qualcuno, per uno scopo da raggiungere.

Una persona gelosa davanti all'evidenza che uno le ha rubato l'oggetto d'amore, avverte odio e un desiderio di colpirlo e può giungere all'estremo di ucciderlo. Una volta realizzato lo scopo, si è consumata la spinta violenta.

Lo stesso meccanismo si attua nella vendetta. Persino nella rapina, si può uccidere per realizzare uno scopo che in questo caso è l'appropriazione di un bene altrui.

Nel momento attuale, sempre meno vediamo casi di violenza finalizzata, mentre dominano quelli della distruttività: che porta a colpire "l'altro da sé", ma nello stesso tempo anche se stesso.

I casi di distruttività rappresentano delle piccole apocalissi: familiari quando uno uccide moglie e figli e poi si suicida. La distruttività segue il "muoia Sansone e tutti i Filistei": distruggiamo tutto.

Una violenza non finalizzata. La nostra è una società distruttiva, una società che fa male e si fa il male.

Una seconda precisazione riguarda il delirio. E' l'errata interpretazione di un evento, di una realtà: una madre che offra un bicchiere d'acqua al proprio figlio che soffre di delirio di persecuzione, si sente non solo rifiutare l'acqua, ma viene aggredita, perché egli pensa che sia 
un gesto teso ad avvelenarlo. Interpreta che quel bicchiere non glielo dia perché lo vede sudato e assetato, ma per ucciderlo. E in questa interpretazione rifiuta la cortesia e reagisce aggressivamente, come per difendersi.

\section{LA DIAGNOSI PSICHIATRICA}

Non c'è dubbio che in questo personaggio il delirio è presente, come idea guida, almeno nei giorni che precedono questa tragedia. E si prepara per compiere quell'azione in modo preciso: aspetta il momento opportuno, compie una prova nel volo di andata per essere sicuro di compiere in maniera efficace la manovra necessaria.

Un delirio di onnipotenza, e ne dà un'anticipazione all'ex fidanzata, per dimostrare che è forte, che vale. Un delirio eroico, per compiere ciò che nessuno farebbe.

E riporta all'eroe greco che deve morire; solo con la morte assume la caratteristica dell'eroe.

Un caso di sicura patologia, una mente fortemente malata, probabilmente egli soffre di un disturbo bipolare, ma certamente nel periodo in cui lo valutiamo è nella fase maniacale.

E nella maniacalità c'è il delirio, ne è il pilastro costituente.

Non è sostenibile l'ipotesi che egli in questa maniera realizzi la volontà di suicidio, e quindi che si trovi in una condizione depressiva, perché se uno vuole suicidarsi non ha bisogno di tutto questo apparato, basta aprire una finestra e buttarsi.

In Lubitz c'è la piena consapevolezza di un gesto che ha un significato del tutto particolare, e che pone il protagonista nella Storia.

Un malato bipolare, in fase di maniacalità guidato alla realizzazione precisa di un gesto che voleva compiere ed ha atteso da giorni il momento per farlo.

La maniacalità è lucida e riesce a portare a termine programmi in maniera ossessiva e puntuale.

Ciò accade all'interno di un problema psicopatologico che conduce il paziente dentro la distruttività.

E' l'eroe che vuole cancellare il mondo, che non avverte il dolore di chi diventa vittima, poiché egli stesso è vittima di sé e percepisce quella fine come esaltante.

La distruttività si riallaccia certamente ad una pulsione di morte, 
di cui ha parlato Freud e che sarebbe costitutiva dell'esistenza umana posta tra eros e tanatos, tra creatività e distruzione.

Una distruttività che si vede, certo in misura più contenuta nel frequente comportamento umano che porta a colpire le persone care, quelle senza delle quali noi non sapremmo vivere. Colpendo l'altro si finisce per fare del male a sé.

\section{TRE FATTORI DEL COMPORTAMENTO UMANO} rischi?”

Viene da chiedersi: "è possibile valutare e prevedere questi

Io non mi sento di affermare che è sempre possibile, posso soltanto dire che la disciplina psichiatrica ha dentro di sé, dentro i propri strumenti, la possibilità di farlo.

E' difficile perché per capire il comportamento di ciascuno di noi (normale o folle) occorre tenere conto di tre fattori.

Il primo è dato della biologia, di come il cervello di una persona è strutturato. Una dimensione importante, ma non la sola: si ritornerebbe alle vecchie concezioni positivistiche che non ci hanno aiutato molto.

Il secondo fattore è dato dalle esperienze vissute. E' sulla esperienza che si costruisce una personalità, e quindi serve condurre una analisi di personalità. E non bastano i test (nemmeno il MMPI, il Minnesota Multiphasic Personality Inventory, il più usato). Occorre stabilire una relazione tra paziente e psichiatra per scoprire comportamenti, idee e desideri. Ma anche questo fattore non basta.

Il terzo è dato dall'ambiente in cui quella persone vive e si esprime.

E' possibile conoscere una personalità, ma è complicato e richiede molto tempo per applicare gli strumenti necessari per farlo.

Ecco perché della psichiatria forse la definizione che io più amo è quella che dà G. Colombo: una scienza infelice.

Certo è una scienza, però ha delle complessità enormi quando la si porta sul piano dell'applicazione. Se poi la si mette dentro i tempi abitualmente dati all'analisi di un caso, il possibile diventa irrealizzabile: un paradosso drammatico.

Tengo molto al termine scienza, ci tengo perché credo molto in quella definizione che diede Jean Piaget, lo psicologo ginevrino che ha avuto molta importanza negli studi sullo sviluppo mentale del bambino. 
Nel 1955, quando si discuteva se la psichiatria e la psicoanalisi fossero scienze, lui parlò di Sciences de l'Homme, che dovevano distinguersi da quelle del particulare, e questa volta la definizione è di Gianbattista Vico.

Le scienze dell'uomo hanno come scopo di tenere l'uomo tutto intero, e vuol dire corpo, psiche, e ambiente, inteso non solo geograficamente, ma come relazioni inter-umane, e quindi nel senso della fenomenologia di uomo-mondo.

Con queste precisazioni termino con un paradosso: il sogno di poter essere sicuri o più sicuri nell'evitare questi eventi, si fonda sulla possibilità di dare la responsabilità di guida a dei robot, che non avendo sentimenti, non avendo deliri di grandezza, riescano a muoversi in maniera meccanica e precisamente prevedibile.

Fino a quel momento l'uomo rimane un essere straordinario, ma un essere che sbaglia, e qui a sbagliare c'è un uomo malato e una Compagnia aerea che non sa scegliere il personale professionale per condurre un aereo. Un aereo, non una bicicletta.

\section{DOMANDE}

"Mi ba turbato il binomio tragedia-abominio, cioè la tragedia personale e l'abominio di trascinare con sé gli altri individui. Noi sappiamo dalla letteratura che il caso del giovane Werther (di Goethe) costitui un esempio che fu emulato, e che l'esibizione pubblica del suicidio può diventare un pericolo collettivo, e in questa forbice tra caso psichiatrico e coinvolgimento collettivo a questi livelli di drammaticità, a lei, come esperto della sua disciplina chiedo se ci sono altre possibilità di emulazione, visto che non di atti terroristici stiamo parlando, ma di malati?".

Per il suicidio è stato ampiamente dimostrato che c'è il rischio di emulazione, e che gli esempi finiscono per trascinare. Il libro I dolori del giovane Werther è stato bruciato in piazza Duomo a Milano, proprio per l'effetto che aveva sui giovani. Ma il tema è il suicidio: la condizione in cui una persona decide di uscire di scena. C'è il suicidio del depresso, ma anche quello di persone che sentono di non potere più avere il ruolo che avevano, e quindi è come se avessero perso di significato e vogliono uscire da questo mondo.

$\mathrm{Nel}$ caso che abbiamo trattato si tratta di un atto maniacale eroico. Il termine eroico potrebbe aprire una lunga discussione, ma semplificando occorre ammettere che noi abbiamo un'idea molto positiva dell'eroe, e 
non ci piace tanto la sentenza di Bertolt Brecht: "beati quei popoli che non hanno bisogno di eroi", che vorrebbe dire che tutti in qualche modo allora vivono un piccolo protagonismo, e non hanno bisogno di chi ne compensi la mancanza con gesti eccessivi, con gesti estremi.

In questo caso domina la brutalità perché ci sono tanti morti, ma non è diverso dall'eroe di guerra, in cui la morte è esaltante.

Ricordo il generale Friedrich Wilheim von Paulus che comandava la $6^{\circ}$ Armata a Stalingrado. Quando percepisce che la battaglia sta per essere perduta, fa un discorso ai propri soldati, che io ho analizzato perché è un capitolo di psichiatria. L'ordine di Hitler è che occorreva resistere e il generale dice a un certo punto: "Cani, volete voi vivere in eterno?", "Sì" risposero, "Allora morite tutti in battaglia". Un'affermazione maniacale.

C'è una maniacalità legata al concetto di eroe; ci sono atteggiamenti in cui gli altri sono solo espressione di un'idea: voi potete morire, anzi dovete, perché questo fa parte di un piano.

L'idea è al di sopra della vita, non solo di uno, ma in questo caso di 150 persone.

"Se questo co-pilota fosse per caso sopravvissuto, che cosa la psichiatria suggerirebbe: di impiccarlo, oppure di ricoverarlo in uno ospedale psichiatrico?"

Forse ti sorprenderò, ma io non avrei voglia di curare questo malato, ma se me lo portano, lo faccio, perché è sicuramente un malato.

Temo però che mai si pentirebbe di ciò che ha compiuto. L'eroe non si pente mai, e probabilmente penserebbe che per il fatto di essere vivo, qualcosa non ha funzionato.

Però questo è un malato, e i malati vanno curati.

Qualche volta le nostre cure sono limitate e imprecise, ma spero che la disciplina di cui faccio parte, che ho chiamato, come hai visto, infelice, aumenti il suo statuto scientifico, e so che abbiamo tanta strada da fare.

Oggi ci sono le neuroscienze che ci aiutano nel primo dei tre fattori che ho citato, nella biologia del cervello.

Considera che la follia generalmente è ciclica, non si dice più alla maniera dei positivisti (di Cesare Lombroso, di Scipio Sighele e di Leonardo Bianchi): "se è matto non può fare niente di normale". Sapessi quante persone importanti sono da me considerate matte!. E ciò complica ulteriormente il lavoro della psichiatria. 